\title{
correspondence
}

\section{Transparency in peer review benefits students}

To the Editor - I greatly enjoyed the recent Editorial in the February issue of Nature Materials on transparency in peer review, and welcome the movement by Nature Publishing Group towards publishing anonymous referee reports and editorial-decision documents. I hope this sets a standard for more, if not all, scientific publishers to follow suit.

In this era of Google and Wikipedia, it's common for researchers (that is, graduate students and postdocs) to quickly look up related information for a paper online and take the information that they find at face value as a way to get up-to-speed as fast as possible. Unfortunately, the most easily available information isn't always the best available information. These primary documents will be a treasure for learning the nuances of a paper, for example why they didn't try a particular strategy/reagent/characterization method.
These documents might tell you that they actually did try at a reviewer's request, and that it was futile for one reason or another.

Joel Kelly

Department of Chemistry, University of Alberta, 11227 Saskatchewan Drive, Edmonton, Alberta T6G 2G2, Canada.

e-mail: joel.kelly@ualberta.ca 